\title{
Costs and benefits of neuroimaging research in obsessive-compulsive disorder: time to take stock
}

\author{
Jon E. Grant ${ }^{1 *}$ and Samuel R. Chamberlain ${ }^{2}$
}

\author{
${ }^{1}$ Department of Psychiatry \& Behavioral Neuroscience, University of Chicago, Chicago, Illinois, USA \\ ${ }^{2}$ Department of Psychiatry, University of Cambridge, Cambridge, UK; Cambridge and Peterborough NHS Foundation Trust, Cambridge, UK \\ First published online 7 May 2018
}

Obsessive-compulsive disorder (OCD) is a neuropsychiatric disorder characterized by obsessions and/or compulsions that are distressing, time consuming, or significantly impairing. OCD is the fourth most common psychiatric illness with a lifetime prevalence of $1-3 \%$, and the World Health Organization (WHO) in 2006 identified OCD as a leading global cause of non-fatal illness burden. At this point, with recent mega-analyses of pooled neuroimaging data in OCD (eg, Fouche et al ${ }^{1}$ ), it is timely to consider what has been learned so far, the costs of this incremental knowledge, and future research targets for the field.

OCD is, for many patients, an eminently treatable disorder. With prompt evidence-based intervention, 60-65\% of affected individuals experience symptom reduction and up to $40-50 \%$ experience remission. ${ }^{2}$ The American Psychiatric Association (USA) and the National Institute for Health and Care Excellence (UK) have published guidelines regarding the diagnosis and management of OCD. Individual exposure/response prevention $(\mathrm{EX} / \mathrm{RP})$ is recommended as the first-line psychotherapy intervention for OCD. EX/RP should be delivered weekly or twice weekly for approximately 20-30 hours of total therapy. After the acute treatment, EX/RP should be delivered as monthly "booster" sessions for 3-6 months. Although EX/RP has demonstrated somewhat better response compared to serotonin reuptake inhibitors (SRIs), SRI therapy is also considered a first-line treatment for OCD. The standard of care today for OCD is essentially based on research from the $1970 \mathrm{~s}$, conducted prior to neuroimaging research. Hence, the questions arise as to

* Address for correspondence: Jon E. Grant, JD, MD, MPH, Department of Psychiatry \& Behavioral Neuroscience, University of Chicago, Pritzker School of Medicine, 5841 S. Maryland Avenue, MC 3077, Chicago, IL 60637, USA.

(Email: jongrant@uchicago.edu) what has been learned so far, at what expense, and where researchers and clinicians should focus their attention next.

We conducted a literature search using PubMed to identify all published imaging studies of OCD as of November 20, 2017 (["MRI" or "imaging" or "neuroimaging" or "magnetic" or "PET" or "positron" or "spectroscopy" or "tomography"] + ["obsessive compulsive disorder" or "obsessive" or "compulsive"]). Reference lists from key review papers were also manually screened for additional source papers. We estimated scan costs based on existing literature, and assumed conservative estimates: charge for a CT head was taken as $\$ 300$, and for an MRI head scan $\$ 1000$. Other scan types were estimated at the same as an MRI head, ie, $\$ 1000$. More direct approximation of scan costs (or associated costs) was not feasible because studies rarely reported total scan time per subject or scan-related costs. Also, to our knowledge, there is no available database maintaining accurate costs of scans over time. These costs are indicative only, since multiple scans may be acquired in the same imaging session but used to generate multiple publications (over-estimate of costs). On the other hand, scans were much more expensive years ago, and there are many indirect costs, eg, staffing (under-estimate of costs).

We found that the total expenditure of OCD neuroimaging research studies, in terms of estimated scan costs, equated roughly to $\$ 10$ million US (1984 to present day). The number of OCD scans per year has been approximately around 1000, and this was found to be increasing exponentially. Recently published studies have used mega-analysis (pooling of scan data across multiple research sites) to confirm or refute the existence of brain abnormalities associated with OCD. In a meta-analysis of MRI scans from $~ 1800$ patients and $\sim 1800$ controls, OCD was associated with significantly reduced hippocampus volumes and elevated pallidum volumes, with small effect sizes according to Cohen's D. ${ }^{3}$ In another analysis, this time in $\sim 400$ patients and $\sim 400$ controls, decreased cortical thickness was found in 
patients in frontal, posterior cingulate, middle temporal, and inferior parietal cortices; as well as in the precuneus. ${ }^{1}$ In a meta-analysis of functional neuroimaging studies, OCD (287 patients versus 284 controls) was associated with hyperactivation in the striatum and insula, alongside hypoactivation in prefrontal cortical regions. ${ }^{4}$ Taking the z-scores from the reported regions of maximum group difference in these two studies, our calculations suggest that the effect sizes of these group differences were small $(<0.25$ by Cohen's D). Thus, it appears that OCD is associated with relatively subtle brain abnormalities using mainstream imaging approaches, based on best available evidence; and that this research knowledge has cost around $\$ 10$ million US. The clinical relevance of such small effect sizes has been questioned. $^{5}$

Current first-line treatment approaches for OCD were initially studied in double-blind trials in the $1970 \mathrm{~s}$, and their effectiveness has been confirmed using various meta-analytic approaches. ${ }^{6}$ Assuming a cost of $\$ 150$ US per hour for EX/RP, and a 16-hour treatment package per person, the total estimated expenditure on brain scan costs between 1984 and present day would have funded psychotherapy treatment for $\sim 4000$ patients over the time frame examined (or $>100$ patients per year). Similarly, this amount of funding would have allowed treatment of an even larger number of patients with pharmacotherapy.

Of course, imaging may in the future have benefit for OCD patients, such as understanding of disease mechanisms (including differentiation from other disorders), identification of new neuro-modulatory targets, and/or contribution to treatment algorithms or outcome prediction (eg, see Hoexter et $a l^{7}$ ). Nonetheless, we think it timely for researchers to question whether casecontrol imaging research based purely on brain structure or blood oxygenation level dependent (BOLD) activation is likely to lead to direct patient benefit, especially if divorced from clinical trials. Longitudinal tracking of dimensional as well as categorical measures relevant to psychopathology is a promising route for enhancement of effect sizes and impact of imaging studies. By combining clinical trials with imaging-and other less expensive biomarkers (eg, blood tests, neuropsychological assessment) - it may be that larger effect sizes can be garnered that will be of more direct clinical utility for patients and society at large, which would collectively fund much of this research.

\section{Disclosures}

Jon Grant and Samuel Chamberlain do not have anything to disclose.

\section{REFERENCES:}

1. Fouche JP, du Plessis S, Hattingh C, et al. Cortical thickness in obsessive-compulsive disorder: multisite mega-analysis of 780 brain scans from six centres. Br J Psychiatry. 2017; 210(1): 67-74.

2. Simpson HB, Huppert JD, Petkova E, Foa EB, Liebowitz MR. Response versus remission in obsessive-compulsive disorder. J Clin Psychiatry. 2006; 67(2): 269-276.

3. Boedhoe PS, Schmaal L, Abe Y, et al. Distinct subcortical volume alterations in pediatric and adult OCD: a worldwide meta- and megaanalysis. Am J Psychiatry. 2017; 174(1): 60-69.

4. Norman LJ, Carlisi C, Lukito S, et al. Structural and functional brain abnormalities in attention-deficit/hyperactivity disorder and obsessive-compulsive disorder: a comparative meta-analysis. JAMA Psychiatry. 2016; 73(8): 815-825.

5. McKay D, Abramovitch A, Abramowitz JS, Deacon B. Association and causation in brain imaging: the case of OCD. Am J Psychiatry. 2017; 174(6): 597.

6. Skapinakis P, Caldwell D, Hollingworth W, et al. A systematic review of the clinical effectiveness and cost-effectiveness of pharmacological and psychological interventions for the management of obsessive-compulsive disorder in children/ adolescents and adults. Health Technol Assess. 2016; 20(43): 1-392.

7. Hoexter MQ, Diniz JB, Lopes AC, et al. Orbitofrontal thickness as a measure for treatment response prediction in obsessive-compulsive disorder. Depress Anxiety. 2015; 32(12): 900-908. 